\title{
Relationship between fat distribution and tongue strength in persons with and without obstructive sleep apnea
}

\begin{abstract}
This study examined the tongue strength and endurance of persons with and without OSA using dual energy X-ray absorptiometry (DEXA) for measures of fat distribution. Subjects included 11 persons ( 8 males, 3 females, $M \pm \mathrm{SD}=65.9 \pm 8.4$ ) with OSA (apnea/hypopnea index $[\mathrm{AHI}]>10 / \mathrm{h}$ sleep) and 17 typical, healthy controls (12 males, 5 females, $M \pm \mathrm{SD}=$ $50.9 \pm 20.22)$. Tongue strength measures included maximum anterior and posterior strength, endurance, and strength during speech and swallowing tasks. DEXA was used to calculate total visceral fat $\%$, total neck fat $\%$, and total body fat $\%$. Results showed no significant difference in tongue strength measures based on the presence of OSA. Regression analyses showed that total body fat \% was able to predict tongue strength during speech $\left(\mathrm{R}^{2}=.291\right.$, $\mathrm{F}(1,26)=10.24, p=.004$. $)$ and visceral fat $\%$ was able to predict tongue strength during swallowing $\left(\mathrm{R}^{2}=.214, \mathrm{~F}(1,26)=6.8, p=.015\right)$. Beta coefficients showed that tongue strength increased as fat \%increased ( $\beta=.539, .462)$. Finally, there was no difference in speech or swallowing tongue strength reserves between the groups, and participants used $17 \%$ and $42 \%$ of their strength reserves during speech and swallowing tasks, respectively. Regression analysis of obesity and tongue strength reserves indicate that with each unit increase in total body fat $\%$, speech reserves increased by more than half a percent $(\beta=0.547 ; p=.003)$. Overall results suggest that reduced tongue strength may not be a factor in the pathogenesis of OSA.
\end{abstract}

Keywords: Obstructive sleep apnea, tongue strength, tongue pressure, functional capacity, obesity
Volume 2 Issue 5 - 2018

\author{
Paul M Evitts,' Dalton Nichols, ${ }^{2}$ Kailyn \\ Asbury, ${ }^{2}$ Taylor Westhoff, ${ }^{2}$ Devon A \\ Dobrosielski \\ 'Department of Audiology, Speech-Language Pathology \& Deaf \\ Studies, Towson University, USA \\ ${ }^{2}$ Department of Kinesiology, Towson University, USA
}

\begin{abstract}
Correspondence: Paul M Evitts, Department of Audiology, Speech-Language Pathology \& Deaf Studies, Towson University, Towson, MD 2I 252, USA, Tel 4|0-704-4|3|,
\end{abstract} Email pevetts@towson.edu

Received: August 30, 2018 | Published: November 22, 2018
Abbreviations: DEXA, dual-energy x-ray absorptiometry; IOPI, iowa oral pressure instrument; OSA, obstructive sleep apnea

\section{Introduction}

Obstructive sleep apnea (OSA) syndrome is characterized by the recurrent collapse of the pharyngeal airway during sleep, which requires arousal to reestablish airway patency and resume breathing. Incidence figures suggest that greater than $10 \%$ of the population suffers from a sleep-related disorder with an estimated $4 \%$ suffering from OSA, specifically. ${ }^{1}$ While the cause of OSA is unknown, it is well accepted that obesity is the strongest predictor of OSA. Indeed, an estimated $30 \%$ of obese patients are diagnosed with $\mathrm{OSA}^{2}$ and the prevalence of OSA among morbidly obese patients ranges from 50 $98 \% .{ }^{3,4}$ The pathogenesis of OSA is incredibly complex. This is due in part, to the physiological and anatomical differences between obese and lean patients, as well as anatomical differences between those with and without OSA..$^{5-7}$ Further confounding the research on OSA is the use of BMI as a measure of obesity which may not be reliable. In fact, a systematic review and meta-analysis of 45 studies that used BMI to identify obesity revealed BMI to have low sensitivity and high specificity. ${ }^{8}$ Furthermore, a cross-sectional study of 1,393 adults by Shah and Braverman ${ }^{9}$ found that $39 \%$ of the subjects were classified as non-obese by BMI but were found to be obese by dual-energy $\mathrm{X}$-ray absorptiometry (DEXA).

\section{Tongue strength and OSA}

Tongue strength has also been implicated as a possible contributor in OSA. For example, the genioglossus has been shown to contribute to airway patency during sleep ${ }^{10}$ and tongue strength during the arousal state has been shown to have a negative correlation with obstructive apnea time and index. ${ }^{11}$ Additionally, persons with moderate OSA have been shown to be more susceptible to tongue muscle fatigue. ${ }^{12,13}$ Moreover, tongue strengthening (i.e., oropharyngeal) exercises and hypoglossal nerve stimulation have been shown to reduce the severity and symptoms for persons with moderate OSA. ${ }^{14,15}$ Conversely, there is evidence suggesting that tongue strength may not play a role with OSA. For example, Mortimore ${ }^{16}$ concluded that neither protrusion strength nor fatigability were important factors in sleep apnea. In addition, Eckert ${ }^{17}$ found that persons with OSA had stronger maximal tongue forces than their healthy counterparts.

\section{Tongue strength, OSA and obesity}

Further confounding the role of tongue strength on OSA is the paucity of research on the impact of obesity on tongue strength. This is relevant since obesity has been shown to be a strong predictor of OSA and a high percentage of those with OSA are obese. ${ }^{2}$ Limited data suggests a positive relationship between tongue strength and BMI in typical healthy persons ${ }^{18}$ and obese persons with OSA. ${ }^{13}$ Additional insight on the impact of obesity on strength can be gleamed from the limb literature which generally shows that obesity is associated with reduced muscular function. ${ }^{19}$ However, it may not be appropriate to generalize results from the limb literature as the muscles associated with speech and swallowing have been shown to be unique from the limb muscles in multiple ways, including muscle fiber composition. ${ }^{20}$ Regardless, there is a lack of consensus in the literature on the impact of obesity on tongue strength in persons with and without OSA and additional studies are warranted. Therefore, the aim of the present was therefore to shed light on the relationship among obesity, OSA, and tongue strength. Based on previous research, it was hypothesized that: 1) persons with OSA will exhibit reduced tongue strength and endurance relative to non-OSA persons; 2) obesity will be associated with reduced tongue strength and endurance; and 3) obesity will be predictive of OSA. Clinically, results may provide important insight 
into factors associated with the presence of OSA and if tongue strength may be a therapeutic target for those persons who are obese and have OSA.

\section{Methods}

\section{Subjects}

The study sample was from the Greater Baltimore Metropolitan area and Towson University. The Towson University Institutional Review Board approved the study protocol and each participant provided written informed consent. Exclusion criteria included diagnosis of head/neck cancer, speech disorder, stroke, swallowing disorder, professional wind instrument player, and any major surgery on the head and/or neck. Polysomnography (PSG) was completed with a Resmed Apnealink polysomnograph at home for one night. Resmed Apnealink is a portable multichannel polysomnograph that measures pulse oximetry, nasal airflow, and respiratory effort. Diagnosis of OSA was made in accordance with the American Association of Sleep Medicine (AASM) guidelines as apnea-hypopnea index more than 10 per hour of sleep. Height and weight was measured using standard stadiometer and digital scale. Percentage of fat and lean tissue was measured using a Lunar Prodigy DEXA machine (GE Healthcare, Madison, WI). Participants were positioned centrally on the scanner with the mandible on a vertical plane. Scans were analyzed with the encore software (version 14.0). Defined standard regions included trunk, android, and gynoid. Further, a customized neck region was defined by incorporating the midpoint of the chin outward to the soft tissue outline of the head, the first bony landmark in link with the scapula, the highest point of the rib cage. Tongue strength measures were collected using the Iowa Oral Pressure Instrument (IOPI Medical, Redmond, WA). The device was calibrated prior to data collection for each subject per the manufacturer's guidelines. Protocol and order for tongue strength measures were based on other research and included: max posterior (x3), max anterior (x3), tongue endurance (x1), swallow max pressure (x3) and speech max pressure (x6). ${ }^{21-23}$ Participants were given a five-minute break after the tongue endurance task prior to the swallowing and speech tasks.

\section{Statistical analysis}

Descriptive statistics were collected for all outcome measures. Since the first research question involved multiple quantitative dependent variables and a single categorical independent variable, a one-way MANOVA was conducted. ${ }^{24}$ The second and third research questions involved multiple quantitative dependent and independent variables, thus multiple regressions were conducted. ${ }^{24}$ Statistical analyses were performed using IBM SPSS (version 24).

\section{Results \\ Descriptive}

Subjects with OSA (AHI $\geq 10 / \mathrm{hr})$ included eight males and three females $(M \pm \mathrm{SD}=65.9 \pm 8.4)$. Based on AHI guidelines from the American Academy of Sleep Medicine, four participants were diagnosed with moderate $\mathrm{OSA}(M \pm \mathrm{SD}=19 \pm 4.03)$ and two participants were diagnosed with severe OSA $\left(M_{-} \mathrm{SD}=43.73 \pm 8.44\right)$. Eight participants were diagnosed with mild OSA $(M \pm S D=10.44 \pm 2.47)$. Subjects with $\mathrm{AHI}<10$ were ultimately classified as non-OSA. Typical healthy subjects included 12 male and 5 female $\left(M_{ \pm} \mathrm{SD}=50.9 \pm 20.22\right)$. Descriptive statistics are provided in Table 1. An independent samples t-test showed none of the differences between the measures to be significant with $p$ values ranging from .057 (total neck fat \%) to .895 (max anterior tongue strength) (Table1).
Table I Mean values of results of DEXA scan and tongue strength measures

\begin{tabular}{lllll}
\hline & Min & Max & Mean & SD \\
\hline OSA AHI & II.I & 49.7 & 20.36 & $12.5 \mathrm{I}$ \\
non OSA AHI & 0 & 8.47 & 3.08 & 2.6 \\
OSA BMI & 19.2 & 42.1 & 28.81 & 6.15 \\
non BMI & 21.5 & 37.7 & 30.83 & 4.39 \\
OSA total body fat \% & 25.99 & 51.3 & 35.5 & 8.55 \\
non total body fat \% & 19.1 & 48.78 & 33.51 & 7.7 \\
OSA total HN \% & 1.89 & 7.51 & 5.14 & 1.62 \\
non total HN \% & 2.87 & 8.9 & 4.71 & 1.38 \\
OSA visceral fat \% & 0 & 8.75 & 4.51 & 2.79 \\
non visceral fat \% & 0 & 5.9 & 2.51 & 2.06 \\
OSA max posterior & 2 & 54 & 29.85 & 15.12 \\
non max posterior & 2 & 80.33 & 33.59 & 19.64 \\
OSA max anterior & 39.67 & 65.33 & 53.45 & 8.86 \\
non max anterior & 19 & 88.33 & 58.92 & 15.16 \\
OSA swallow & 1.67 & 54.33 & 14.33 & 14.34 \\
non swallow & 3.33 & 29 & 12.24 & 6.81 \\
OSA speech & 3.67 & 26.33 & 10.47 & 6.98 \\
non speech & 0 & 23.17 & 8.09 & 4.75 \\
OSA endurance & 11 & 30 & 21.27 & 5.83 \\
non endurance & 8 & 62 & 28.58 & 15.61 \\
\hline
\end{tabular}

Note: OSA, obstructive sleep apnea; HN, head and neck; SD, standard deviation; $\mathrm{AHI}$, apnea/hypopnea index. Tongue strength units were measured in $\mathrm{k} / \mathrm{Pa}$

\section{Tongue strength and OSA}

A one-way MANOVA was conducted using tongue strength and endurance measures as the dependent variables and the presence of OSA as the independent variable to examine the relationship between measures of tongue strength and OSA. Table 2 shows none of the tongue strength measures had a significant main effect on the presence of OSA. Partial eta squared values were all below 108 indicating that less than $11 \%$ of the variance associated with tongue strength was associated with the presence of OSA (Table 2).

Table 2 Results of the one-way MANOVA for tongue strength and presence of OSA

\begin{tabular}{lllll}
\hline $\begin{array}{l}\text { Tongue } \\
\text { strength } \\
\text { measure }\end{array}$ & F & Significance & $\begin{array}{l}\text { Partial eta } \\
\text { squared }\end{array}$ & $\begin{array}{l}\text { Observed } \\
\text { power }\end{array}$ \\
\hline Max Posterior & 0.196 & 0.662 & 0.008 & 0.196 \\
Max Anterior & 1.216 & 0.281 & 0.046 & 1.216 \\
Swallow & 0.428 & 0.519 & 0.017 & 0.428 \\
Speech & 1.057 & 0.314 & 0.041 & 1.057 \\
Endurance & 3.022 & 0.094 & 0.108 & 3.022 \\
Speech reserve & 1.315 & 0.262 & 0.05 & 1.315 \\
Swallow reserve & 0.081 & 0.779 & 0.003 & 0.081 \\
\hline
\end{tabular}

Note: OSA, obstructive sleep apnea 


\section{Tongue strength and obesity}

Results of the stepwise multiple regression analyses indicated that obesity predicted two of the tongue strength measures: mean speech pressure and mean swallow pressure. Specifically, model estimates suggest that with each unit increase in total body fat \%, tongue strength during speech increased by more than half a kPA unit $(\beta=0.539 ; p=.004)$. Overall model fit statistics indicated that total body fat \%accounted for $29 \%$ of the observed variance $\left(\mathrm{R}^{2}=.291\right.$, $\mathrm{F}(1$, 26) $=10.24, p=.004)$. Likewise, regression results indicated that with each percentage point increase in visceral fat, tongue strength during swallowing increased by nearly half a kPA unit $(\beta=0.462 ; p<.015)$. Overall model fit suggested that visceral fat $\%$ accounted for $21 \%$ of the variance $\left(\mathrm{R}^{2}=.214, \mathrm{~F}(1,26)=6.8, p=.015\right)$. Considered together, total body fat \%and visceral fat \% were found to be significant predictors of tongue strength during speech and swallowing, with each predictor accounting for approximately one-fifth of the variation in tongue strength across subjects.

\section{Obesity and OSA}

Table 3 Tongue strength reserves for speech and swallowing tasks

\begin{tabular}{llllllll}
\hline & Mean speech & Mean max anterior & Speech reserve (\%) & Mean swallow & $\begin{array}{l}\text { Mean max } \\
\text { posterior }\end{array}$ & Swallow reserve (\%) \\
\hline OSA & $10.47(7.0)$ & $53.45(8.8)$ & 20 & $14.33(14.33)$ & $29.84(15.1)$ & 48 \\
non OSA & $8.09(4.8)$ & $58.92(15.1)$ & 15 & $12.24(6.8)$ & $33.59(19.6)$ & 36 \\
Overall mean & $9.02(5.7)$ & $56.77(13.1)$ & 17 & $13.06(10.2)$ & $32.12(17.8)$ & 42 & \\
\hline
\end{tabular}

Note: Tongue strength units measured in $\mathrm{kPa}$. Speech reserve calculated by mean speech or swallow pressure/mean maximum anterior (speech) or posterior (swallow) pressure. Standard deviations are in parentheses.

Table 4 Results of the stepwise multiple regression analysis for speech reserves

\begin{tabular}{lllllll}
\hline & \multicolumn{1}{c}{ Model I } & \multicolumn{3}{c}{ Model 2 } \\
Variable & B & SE B & $\beta$ & B & SE B & $\beta$ \\
\hline Constant & -0.1 & 0.084 & & -0.166 & 0.085 & \\
Total body fat \% & 0.008 & 0.002 & $0.547^{*}$ & 0.008 & 0.002 & $0.573^{*}$ \\
Visceral fat \% & & & & 0.015 & 0.007 & $0.335^{*}$ \\
$\mathrm{R}^{2}$ & & & & & 0.41 & \\
F for change in R2 & & 0.299 & & & $4.53^{*}$ & \\
\hline
\end{tabular}

Note: Dependent variable $=$ speech reserve. Variables entered $=$ BMI, total head and neck fat \%, total body fat \%, and visceral fat \%
To determine the relationship between tongue strength reserves and measures of obesity, a multiple regression was calculated. Table 4 shows two different models of obesity measures that were predictive of speech reserves. The second regression model which included total body fat $\%$ and visceral fat $\%$ was able to predict $41 \%$ of the overall variance associated with speech reserve. Beta values indicate that with each unit increase in total body fat $\%$, speech reserves increased by more than half a percent $(\beta=0.547 ; p=.003)$. Furthermore, beta values for the second model indicate that for each unit increase in visceral fat $\%$, speech reserves increased by $0.3 \%$ (Table 4 ).

\section{Discussion}

The purpose of this study was to investigate the relationship among tongue strength, OSA, and obesity. Overall, results suggest that tongue strength and endurance are not predictors of OSA but that total body fat $\%$ and visceral fat $\%$ were predictive of tongue pressures during more functional activities such as speech and swallowing. Results also suggest that as total body fat and visceral fat increase, subjects use a higher proportion of their overall maximum tongue strength (reserve) during speech
Results of the stepwise multiple regression analyses indicated that visceral fat \% predicted AHI. Specifically, model estimates suggest that with each unit increase in visceral fat $\%$, AHI increased by .38 $(\beta$ $=0.381 ; p=.05)$. Overall model fit statistics indicated that visceral fat $\%$ accounted for nearly $15 \%$ of the observed variance $\left(\mathrm{R}^{2}=.145, \mathrm{~F}(1\right.$,

\section{Tongue strength reserves}

It was also of interest to use the existing results on tongue strength measures to determine the total percent of maximum function for both speech and swallowing activities. Similar to other researchers, the percent of tongue strength by function, or the amount of reserve, was calculated for speech and swallowing functions (speech reserve $=$ mean speech $/ \max$ anterior; swallow reserve $=$ mean swallow $/ \mathrm{max}$ posterior). ${ }^{22}$ Results are provided in Table 3. A one-way ANOVA showed no significant difference between the two groups in speech reserve values, $\mathrm{F}(1,26)=1.32, p=.262$, or swallowing reserve values, $\mathrm{F}(1,26)=.081, p=.779$ (Table 3$)$. 26) $=4.25, p=.05)$.

\section{Tongue strength and OSA}

Current results showed that none of the tongue strength measures were associated with the presence of OSA. Descriptively, persons with OSA generated higher tongue pressure values during functional activities (i.e., speech and swallowing) than their typical counterparts but not during tasks involving maximum pressures or endurance tasks. While none of these comparisons were significantly different between the two groups, the presence of a trend for persons with OSA to have higher mean values during those functional tasks is notable and is consistent with other similar research. ${ }^{16,17}$ The absence of a significant difference in tongue strength is, however, in contrast to research showing the benefits of oropharyngeal exercises ${ }^{15}$ or hypoglossal nerve stimulation ${ }^{25}$ on OSA. Muscle groups targeted with specific oropharyngeal exercises include the soft palate, tongue, and face which may explain the discrepant results. Since numerous muscle groups are targeted, it is difficult to determine the isolated role of tongue strength in the improved AHI scores.

Results also showed a trend for persons with OSA to have reduced endurance, representing nearly a $30 \%$ magnitude of difference. When 
coupled with a trend of higher maximum strength values, results suggest that fatigability rather than maximal strength may be an issue for persons with OSA. Similar results were reported by Eckert. ${ }^{17}$ who showed that persons with OSA had greater maximal tongue protrusion force than controls but significantly reduced endurance. Thus, the beneficial effects of exercises for OSA may not be related to overall strength gains per se, but more so related to improvements in endurance. Clearly, larger sample sizes are required before generalizations can be made but the aggregated results suggest that the overall strength of the genioglossus may not be a factor in OSA.

\section{Tongue strength and obesity}

Overall results of this study showed that increased total body fat $\%$ and visceral fat $\%$ resulted in increased tongue strength during speech and swallowing tasks, respectively. While limited, other studies have shown similar results $\mathrm{Li}^{13}$ and Ulrich Sommer. ${ }^{18}$ Additionally, other research comparing the tongue strength of active and non-active adults shows that active adults have significantly stronger anterior and posterior maximal values. ${ }^{26}$ Existing data coupled with the current results suggest that increased fat content in the oropharyngeal region may be immune to reduced strength as seen with the limbs. That increased fat in the tongue may not be associated with reduced strength may be either the result of inherent differences in muscle physiology ${ }^{20}$ or attributed to increased use. Although obesity is associated with increased sedentary behavior, ${ }^{27}$ this behavior may not be consistent with the speaking and swallowing musculature. Simply put, persons who are obese may have stronger tongues during functional activities (e.g., speech, swallowing) due to increased use. In fact, Ulrich Sommer ${ }^{18}$ postulated that the increased tongue strength often found with obesity was related to increased exercise via increased food intake. Hence the positive correlation between BMI and tongue strength. When discussing obesity and muscular function, it is important to distinguish between absolute maximum strength and relative weakness. It is commonly accepted that obesity is associated with increased absolute maximum strength due to increased muscle size. However, when body mass is factored, persons who are obese have reduced strength relative to their typical-weight counterparts. ${ }^{28}$ This is evidenced by multiple studies on limb strength showing higher absolute strength values for persons who are obese, but significantly weaker muscular function when normalized for body mass Janssen. ${ }^{29}$ This phenomenon may be partially explained by increased adiposity and reduced muscle density. ${ }^{19}$

\section{Obesity and OSA}

Current results showed that visceral fat $\%$ was a significant predictor of AHI. This is in concert with the multitude of studies showing the strong relationship between obesity and OSA Bruno. ${ }^{30}$ While complex, it has been suggested that increased adiposity in the trunk reduces chest compliance and has a negative impact on functional residual capacity. ${ }^{4}$ Although total neck fat $\%$ was not predictive of AHI in the current study using the regression model, there was a weak-moderate correlation between AHI and total neck fat $\%(r=.344)$. Other studies have suggested that increased fat in the oropharyngeal regions contribute to creating a smaller upper airway and thus may play a role in the pathogenesis of OSA. ${ }^{31}$ In fact, persons with OSA have been shown to have significantly more neck fat mass than controls while having a significant decrease in fat free mass in the oral region..$^{30}$ One possible explanation for neck fat \% not being a significant predictor in the current study may be how the percent of neck fat was extracted from the DEXA results. In addition, the fact that BMI was not a predictor of AHI is noteworthy and contributes to the increasing body of evidence showing that BMI may not be a reliable measure of obesity ${ }^{8,9}$ and that future research should include more accurate indices of obesity.

\section{Tongue strength reserves}

Descriptive results showed that persons with OSA were required to use a higher percentage of their total tongue strength (reserve) than their typical counterparts for speaking and swallowing. This relationship between reserve and strength is related to functional capacity, or the performance requirements of daily tasks Booth, ${ }^{32}$ Van Ravenhorst-Bell ${ }^{26}$ note that increased functional capacity results in greater reserve capacity. While there was not a statistically significant difference in strength reserves between OSA groups, increased total body fat was associated with increased reserve. Total body and visceral fat $\%$ also accounted for $41 \%$ of the variance associated with speech strength reserves. Reserve values observed were relatively consistent with previous research. Specifically, Neel and Palmer ${ }^{22}$ reported that speakers used an overall mean of $17 \%$ of their total tongue strength during a similar speech task and Youmans ${ }^{33}$ found that women and men used $63 \%$ and $49 \%$, respectively, of their total maximum pressures during a swallowing task.

Because of the strong relationship between OSA and obesity, results indicate that obesity may also be associated with reduced functional capacity for with regard to tongue strength. That is, the overall strength of the tongue may be adequate, but functional capacity in relationship to strength reserves is at issue. The fact that persons with OSA had higher mean values of tongue strength reserves and that obesity was predictive of strength reserves has important clinical implications. For example, a high percentage of persons with OSA are obese and obesity is associated with greater health complications. When complications arise, persons with OSA may have less reserve to recruit for those functional oral activities such as speaking and swallowing. More research on tongue strength reserves in both persons who are obese and/or who have OSA will hopefully help elucidate this relationship and may provide important clinical insight into therapeutic targets.

\section{Limitations}

There are multiple limitations to this study which make generalization to the entire OSA population difficult. First, there was a small sample size of persons with OSA and their typical healthy counterparts. This includes a small number of persons with varying degrees of OSA. Clearly, increased sample sizes including a greater representation of severity levels of OSA would permit greater generalization of the data. Second, by subtracting head fat $\%$ and only reporting total neck fat $\%$, the use of that outcome measure may not reflect the role of increased adiposity in the palatal and pharyngeal regions.

\section{Conclusion}

Overall results of the study suggest that tongue strength and endurance are not factors in the pathogenesis of OSA. In addition, results also suggest that increased adiposity is associated with increased tongue strength during functional oral activities such as speaking and swallowing. This stands contrary to the bulk of the limb literature showing a strong association between obesity and reduced muscular strength. Finally, results suggest that persons who are obese and who have OSA may be using a higher proportion of their overall tongue strength (i.e., reserves) than their typical, healthy counterparts. In aggregate, results imply that targeting tongue strength for the treatment of OSA may not be appropriate. 


\section{Acknowledgements}

The authors would like to thank Melissa Sechler MS., CCC-SLP for assistance with data collection on an earlier version of this study. Portions of the data were presented at the American Speech-Language and Hearing Association (ASHA) annual convention Denver, CO 2015. Author Contributions: PME and DAD defined the study. PME and KA prepared the manuscript. PME conducted the data analysis and interpretation. DN, KA, and TW recruited subjects and collected data.

\section{Conflict of interest}

Disclosure Statement: The authors report no financial or nonfinancial conflicts of interest. In addition, this research did not receive any specific grant from funding agencies in the public, commercial, or not-for-profit sectors.

\section{References}

1. Resta O, Barbaro MP, Giliberti T, et al. Sleep related breathing disorders in adults with Down Syndrome. Down Syndr Res Pract. 2003;8:115-119.

2. Brown LK. A waist is a terrible thing to mind: central obesity, the metabolic syndrome, and sleep apnea hypopnea syndrome. Chest. 2002;122:774-778.

3. Fisher D, Pillar G, Malhotra A, et al. Long term follow-up of untreated patients with sleep apnea syndrome. Respir Med. 2002;96:337-343.

4. Pillar G, Peled K, Katz N, et al. Predictive value of specific risk factors, symptoms and signs, in diagnosing obstructive sleep apnea and its severity. J Sleep Res. 1994;3:241-244.

5. Ferguson KA, Takashi O, Lowe AA, et al. The relationship between obesity and craniofacial structure in obstructive sleep apnea. Chest. 1995;2:375-381

6. Nashi N, Kang S, Barkdull, GC, et al. Lingual fat at autopsy. Laryngoscope. 2007;117:1467-1473.

7. Tsuchiya M, Lowe AA, Pae E, et al. Obstructive sleep apnea subtypes by cluster analysis. Am J Orthod Dentofac Orthop. 1992;101:533-542.

8. Okorodudu DO, Jumean MF, Montori VM, et al. Diagnostic performance of body mass index to identify obesity as defined by body adiposity: a systematic review and meta-analysis. Intl J Obes. 2010; 34:791-799.

9. Shah NR, Braverman ER. Measuring adiposity in patients: The utility of body mass index (BMI), percent body fat, and leptin. PloS one. 2012; 7:e33308.

10. Remmers JE, deGroot WJ, Sauerland EK, et al. Pathogenesis of upper airway occlusion during sleep. $J$ Appl Physiol Environ Exerc Physiol. 1978:44:931-938

11. Kanezaki M, Ogawa T, Izumi T. Tongue Protrusion strength in arousal state is predictive of the airway patency in obstructive sleep apnea. Tohoku J Exp Med. 2015;236:241-245.

12. Blumen MB, de La Sota AP, Quera-Salva MA, et al. Tongue mechanical characteristics and genioglossus muscle EMG in obstructive sleep apnoea patients. Resp Phys \& Neurob. 2004;140:155-164.

13. Li WY, Gakawaya S, Saey D,Sériès F. Assessment of tongue mechanical properties using different contraction tasks. J Appl Physio (1985). 2017;123(1):116-125.

14. Eastwood PR, Barnes M, Walsh JH, et al. Treating obstructive sleep apnea with hypoglossal nerve stimulation. Sleep. 2011;34:1479-1486.
15. Guimarães KC, Drager LF, Genta PR, et al. Effects of oropharyngeal exercises on patients with moderate obstructive sleep apnea syndrome. Am J Respir Crit Care Med. 2009;179:962-966.

16. Mortimore IL, Bennett SP, Douglas NJ. Tongue protrusion strength and fatigability: relationship to apnoea/hypopnoea index and age. J Sleep Res. 2000;9:389-393.

17. Eckert DJ, Lo YL, Saboisky JP, et al. Sensorimotor function of the upper-airway muscles and respiratory sensory processing in untreated obstructive sleep apnea. J ApplPhysiol. 2011;111:1644-1653.

18. Ulrich Sommer J, Birk R, Hörmann K, et al. Evaluation of the maximum isometric force of healthy volunteers. Eur Arch Otorhinolaryngol.2014;271(11):3077-3084.

19. Goodpaster BH, Carlson CL, Visser M, et al. Attenuation of skeletal muscle and strength in elderly: The health ABC study. J Appl Phys. 2001;90:2157-2165.

20. Kent, R. The uniqueness of speech among motor systems. Clin Ling Phon. 2004;18(6-8):495-505.

21. Crow HC, Ship A. Tongue strength and endurance in different aged individuals. J Gero Series A. 1996;51(5):M247-250.

22. Neel AT, Palmer PM. Is tongue strength an important influence on rate of articulation in diadocho kinetic and reading tasks? J Sp Lang Hear Res. 2012;55(1):235-246

23. Vanderwegen J, Guns C, Van Nuffelen G, et al. The influence of age, sex, bulb position, visual feedback, and the order of testing on maximum anterior and posterior tongue strength and endurance in healthy Belgian adults. Dysphagia. 2013;28(2):159-166.

24. Mertler CA,Vannatta RA. Advanced and multivariate statistical methods. 2nd ed. GlenDale(CA): Pyrczak publishing; 2002.

25. Strollo PJ, Jr Soose RJ, Maurer JT, et al. Upper-airway stimulation for obstructive sleep apnea. $N$ Engl J Med. 2014;370:139-149.

26. Van Ravenhorst-Bell HA, Mefferd AS, Coufal KL, et al. Tongue strength and endurance: Comparison in active and non-active young and older adults. Int J Sp Lang Path. 2017;19(1):77-86.

27. Maher CA, Mire E, Harrington DM, et al. The independent and combined associations of physical activity and sedentary behavior with obesity in adults: NHANES 2003-06. Obes (SilvSpr.) 2013;21(12):E730-737.

28. Tomlinson DJ, Erskine RM, Morse CI, et al. The impact of obesity on skeletal muscle strength and structure through adolescence to old age. Biogerontology. 2016;17(3):467-483.

29. Janssen I, Heymsfield SB, Wang ZM, et al. Skeletal muscle mass and distribution in 468 men and women aged 18-88 yr. J Appl Physio(1985). 2000;89(1):81-88.

30. Bruno E, Alessandrini M, Napolitano B, et al. Dual-energy X-ray absorptiometry analysis of body composition in patients affected by OSAS. Eur Arch Otorhinolaryngol. 2009;266(8):1285-1290.

31. Schwab RJ, Pasirstein M, Pierson R, et al. Identification of upper airway anatomic risk factors for obstructive sleep apnea with volumetric magnetic resonance imaging. Am J Respir Crit Care Med. 2003;168(50):522-530.

32. Booth FW, Laye M J, Roberts MD. Lifetime sedentary living accelerates some aspects of secondary aging. $J$ Appl Phys (1985). 2011;111(5):14971504.

33. Youmans S, Youmans G, Stierwalt J. Differences in tongue strength across age and gender: Is there a diminished strength reserve? Dysphagia. 2009;24(1):57-65. 